\title{
Severe duodenojejunitis in a case of adult-onset Henoch-Schönlein purpura
}

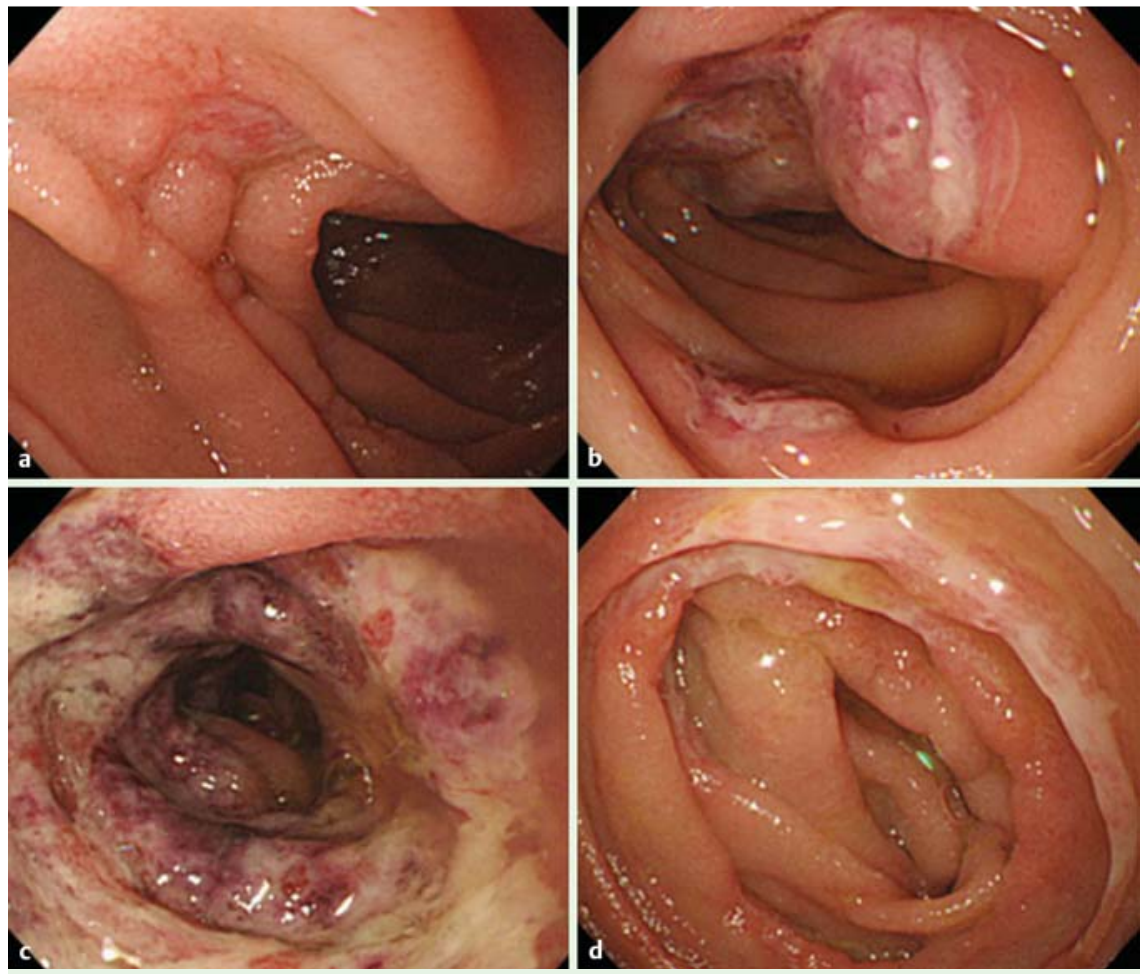

Fig. 1 a-d Findings of single-balloon endoscopy of the duodenum and upper jejunum in a patient with adult-onset Henoch-Shönlein purpura. a A small shallow ulcer in the second portion of the duodenum. $\mathbf{b}$ Multiple round ulcers with elevated surroundings in the third portion of the duodenum. c Segmental 5-cm-long ulcer with petechiae in the third portion of the duodenum. $\mathbf{d}$ Circular ulcer in the upper ileum.

A 54-year-old woman with a 2-week history of left upper abdominal pain was admitted to our hospital. Joint pain was not reported. Abdominal CT scan revealed wall thickening from the third portion of the duodenum to the upper jejunum, with surrounding lymphadenopathy. Single-balloon endoscopy (SBE) showed edema, hyperemia, and ulcers from the second portion of the duodenum to the upper jejunum, while the third portion of the duodenum showed more severe damage ( $\bullet$ Fig. 1 ).

Biopsy specimens showed deposition of immunoglobulin $\mathrm{A}$ in the vascular wall (๑ Fig. 2a).

Total colonoscopy demonstrated aphthous lesions in the transverse colon. Palpable purpura appeared on both lower extremities 5 days after admission. Perivascular granulocyte infiltration and fragmented nuclei were found ( $\mathbf{F i g} . \mathbf{2} \mathbf{b}$ ). The level of serum factor XIII was 26\% (normal: $\geq 70 \%$ ), supporting the diagnosis of HenochSchönlein purpura (HSP). Oral administration of $40 \mathrm{mg}$ prednisolone was initiated. The abdominal pain disappeared on the day after prednisolone administration. SBE performed 2 weeks after corticosteroid therapy showed remarkably ameliorated duodenal and jejunal lesions.

Gastrointestinal symptoms occur in 78.2\% of patients with HSP, and of these abdominal pain is the most common symptom [1]. Esophagogastroduodenoscopy findings demonstrated the second portion of the duodenum to be the most frequently involved part of the upper gastrointestinal tract in patients with HSP [1,2]. Using video capsule endoscopy, petechiae/red-

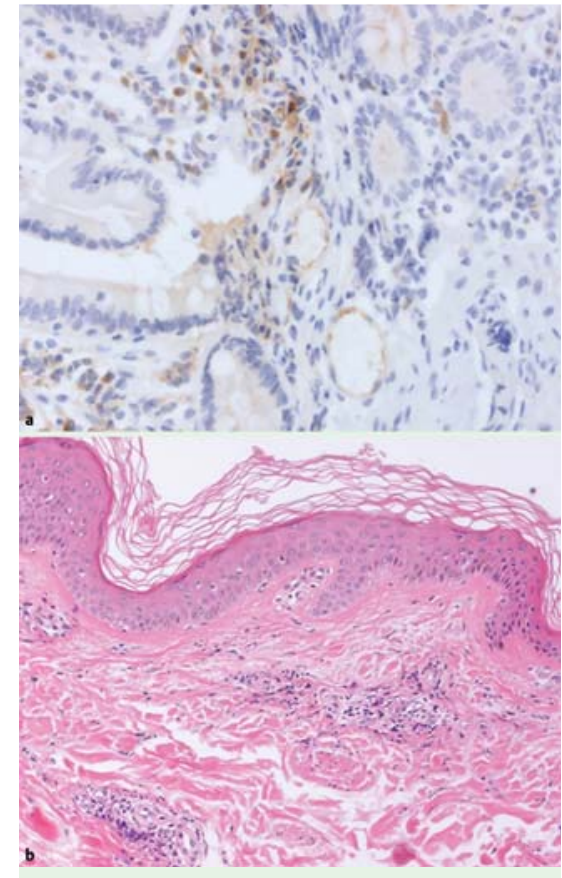

Fig. 2 a Immunohistological staining of the duodenal biopsy specimen demonstrates vascular deposition of immunoglobulin A. b Hematoxylin-eosin staining of the skin biopsy specimen demonstrates perivascular granulocyte accumulation.

ness has been found throughout the small intestine of patients with HSP [3]. The present case demonstrated a remarkable difference in the severity of mucosal injuries between the second and third portions of the duodenum, which was detected by SBE, but would have been impossible to identify by conventional esophagogastroduodenoscopy.

We conclude that SBE should be considered in patients with HSP to evaluate duodenal and upper jejunal injury, to assess the severity of mucosal damage, and to determine the appropriate therapeutic strategy.

Endoscopy_UCTN_Code_CCL_1AB_2AZ_3AC

Competing interests: None 


\section{H. Matsumoto, N. Oshitani, K. Aomatsu,} I. Suwa

Department of Gastroenterology, Izumiotsu Municipal Hospital, Izumiotsu City, Osaka, Japan

\section{References}

1 Zhang Y, Huang X. Gastrointestinal involvement in Henoch-Schönlein purpura. Scand J Gastroenterol 2008; 43: 1038-1043

2 Esaki M, Matsumoto T, Nakamura $S$ et al. GI involvement in Henoch-Schönlein purpura. Gastrointest Endosc 2002; 56: 920-923

3 Ichikawa R, Hosoe N, Imaeda $\mathrm{H}$ et al. Evaluation of small-intestinal abnormalities in adult patients with Henoch-Schönlein purpura using video capsule. Endoscopy 2011; 43: $162-163$

\section{Bibliography}

DOI $10.1055 / \mathrm{s}-0030-1256957$

Endoscopy 2011; 43: E420 - E421

(c) Georg Thieme Verlag KG Stuttgart · New York . ISSN 0013-726X

\section{Corresponding author}

\section{N. Oshitani}

Department of Gastroenterology

Izumiotsu Municipal Hospital

16-1, Shimojuou-machi

Izumiotsu City

Osaka 595-3027

Japan

Fax: +81-725-32-8056

endoscope@hosp-ozu-osaka.jp 